\title{
Cognitive impairment and Alzheimer's disease: Links with oxidative stress and cholesterol metabolism
}

\author{
Alejandra Sekler ${ }^{1,2}$ \\ José $M$ Jiménez ${ }^{2}$ \\ Leonel Rojo ${ }^{2}$ \\ Edgard Pastene ${ }^{3}$ \\ Patricio Fuentes ${ }^{4}$ \\ Andrea Slachevsky ${ }^{4}$ \\ Ricardo B Maccioni ${ }^{1,2}$. \\ 'Center of Cognitive Neurosciences, \\ International Center for Biomedicine \\ (ICC), Santiago, Chile; ${ }^{2}$ Laboratory \\ of Cellular, Molecular Biology and \\ Neurosciences, Faculty of Sciences, \\ Universidad de Chile, Santiago, Chile; \\ ${ }^{3}$ Department of Pharmacy, Faculty of \\ Pharmacy, University of Concepcion, \\ Concepción, Chile; ${ }^{4}$ Unidad de \\ Neurología Cognitiva y Demencias, \\ Servicio de Neurología, Hospital del \\ Salvador, Santiago, Chile
}

\begin{abstract}
Oxidative stress has been implicated in the progression of a number of neurodegenerative diseases, including Alzheimer's disease (AD), Parkinson's disease and amyotrophic lateral sclerosis. We carried out an in-depth study of cognitive impairment and its relationships with oxidative stress markers such as ferric-reducing ability of plasma (FRAP), plasma malondialdehyde and total antioxidative capacity (TAC), as well as cholesterol parameters, in two subsets of subjects, AD patients $(n=59)$ and a control group of neurologically normal subjects $(\mathrm{n}=29)$, attending the University Hospital Salvador in Santiago, Chile. Cognitive impairment was assessed by a set of neuropsychological tests (Mini-Mental State Examination, Boston Naming Test, Ideomotor Praxia by imitation, Semantic Verbal Fluency of animals or words with initial A, Test of Memory Alteration, Frontal Assessment Battery), while the levels of those oxidative stress markers and cholesterol metabolism parameters were determined according with standard bioassays in fresh plasma samples of the two subgroups of patients. No significant differences were observed when the cholesterol parameters (low-, high-density lipoprotein, total cholesterol) of the AD group were compared with normal controls. Interestingly, a correlation was evidenced when the levels of cognitive impairment were analyzed with respect to the plasma antioxidant capacity (AOC) of patients. In this context, the subset of subjects exhibiting cognitive impairment were divided into two subgroups according with their Global Dementia Scale performance: a subgroup with mild AD and a subgroup with moderate to severe AD. Significant differences in AOC were found between subgroups. The different correlations between cognitive impairment of subgroups of subjects with the oxidative stress profile are discussed in the context of AD pathogenesis.
\end{abstract}

Keywords: oxidative stress, molecular biomarkers, cholesterol parameters, cognitive impairment, Alzheimer's patients

\section{Introduction}

Alzheimer's disease (AD) is the most common cause of dementia nowadays. This disease is characterized by the formation of protein aggregates in the human brain, namely paired helical filaments composed of hyperphosphorylated tau and senile plaques of $A \beta$ (Maccioni et al 2001). The pathogenesis of AD and other neurodegenerative disorders has been related with oxidative stress, which might be responsible for the resulting dysfunction and death of neuronal cells. Oxidative stress is the result of the unregulated production of reactive oxygen (ROS) or nitrogenated species, which alters the structure of proteins, lipids and nucleic acids (Perry et al 2000; Barnham et al 2004). These investigations have suggested several hypotheses on the mechanisms of pathogenesis potentially implicated in AD as related with oxidative damage. Studies have also pointed to relationships between progression of dementia and neurodegenerative disorders with the increase in oxidative stress biomarkers (Kikuchi et al 2002; Nunomura et al 2007). 
Moreover, evidence exists that cholesterol is linked to the development of AD (Rojo et al 2006). Hypercholesterolemia is an early risk factor for the development of amyloid pathology, and longitudinal, population-based studies demonstrated that cholesterol is associated with $\mathrm{AD}$ in the later lifespan (Kivipelto et al 2001). On the other hand, ApoE participates in the transport of cholesterol and other lipids in the blood stream and in the cerebrospinal fluid (CSF). This protein is expressed by three major alleles: $\varepsilon 2, \varepsilon 3$, and $\varepsilon 4$, being the homozygozity for Apoe4, is the most relevant risk factor for AD (Corder et al 1993; Lavados et al 2005). However, its mode of action is still unknown (Goedert and Spillantini 2006). The levels of peroxidation in the brains of $\mathrm{AD}$ patients depended on the ApoE genotype, and is higher when Apoc4 allele is present, while the level of peroxidation was inversely proportional to the concentration of ApoE (Rammassamy et al 1998). This lipid-transport protein is a target for the attack of free radicals, and a correlation exists between ApoE peroxidation and AD (Leininger-Muller et al 1998). Prior studies with patients had confirmed that oxidative stress is clearly associated with cognitive decline (Berr et al 2000), and an oxidative imbalance in AD patients has been proven with different oxidative stress biomarkers (Pulido et al 2005; Guidi et al 2006; Zafrilla et al 2006).

In this article, we analyze the oxidative stress status and some of the cholesterol metabolism parameters from a group of $\mathrm{AD}$ patients with different stages of dementia, as compared with neurologically normal controls. Moreover, we have also attempted to correlate cognitive impairment with changes in cholesterol homeostasis.

\section{Materials and methods}

\section{Subjects}

Eighty-eight subjects in total were included in this study. All the recruited participants were more than 60 years-old and recruited from the Metropolitan area population of Santiago, Chile, genetically composed by around $40 \%$ Amerindians and $60 \%$ Caucasians. The AD patients subset included fiftynine subjects (mean age 76.4 years, SD 6.1, 32 women and 27 men) diagnosed with probable Alzheimer-type dementia according to NINCDS/ADRDA criteria (McKhann et al 1984). This subset was compared with twenty-nine control subjects (mean age 70.7 years, SD 6.9, 20 women and 9 men). The subjects in the control subset were recruited from among elderly relatives with the indicated ages and gender distributions. All patients and control subjects were incorporated into the study after informed consent. Only subjects giving informed consent for themselves or from caregivers, and able to be evaluated neuropsychologically were recruited, thus concentrating the population in the study in 88 subjects. They did not exhibit psychiatric and neurological clinical antecedents. Moreover, none of the participants had any other major medical illness or were taking any medication known to affect oxidative stress markers or the cholesterol parameters. Moreover, caution was taken that individuals recruited in the study were not taking any supplement of vitamin E or C, nor extracts with high content of polyphenols (Vitis vinifera, Ginkgo biloba, Camellia sinensis). The education level of the entire population was also analyzed. For evaluation of cholesterol and oxidative markers parameters, venous blood samples were obtained from each subject under study. Subjects were under fasting condition early in the morning, and determinations were carried our in the separated plasma samples. All the experimental protocols were approved by the Committee on Ethical Issues of the Faculty of Medicine, University of Chile, and all subjects provided informed consent prior to the initiation of the study. In the cases of demented subjects, the informed consent for participation in the study was obtained from their caregivers.

\section{Neuropsychological battery of tests}

Once the subjects were included in the AD or control group, they were subjected after informed consent of their caregivers, a neuropsychological battery of tests. The neuropsychological evaluation consisted of the neuropsychological battery that includes Mini Mental State Examination (MMSE), Grober and Buschke, Global Dementia Scale (GDS) (Reisberg et al 1998), Ideomotor Praxia by imitation (PRAXIAS), Boston Naming Test (BNT) of 12 images, Frontal Assesment Battery (FAB), and Semantic Verbal Fluency of animals or words with initial A (FLU Animals and FLU A). Thus the 88 subjects in the study were properly evaluated and included in all measurements.

\section{Measurement of cholesterol parameters}

The total plasma cholesterol levels were determined using the cholesterol assay (Abbott Diagnostics, Huechuraba, Chile) according to the manufacturer's instructions. The low-density lipoprotein (LDL) cholesterol in human plasma was measured using a MULTIGENT Direct LDL assay (Abbott Diagnostics). In the same way, the quantitation of high-density lipoprotein (HDL) cholesterol in human plasma was employed the Ultra HDL assay (Abbott Diagnosis). The VLDL cholesterol was calculated as the difference between total cholesterol and both HDL and LDL. The index total cholesterol/HDL was calculated with the data obtained of the experimental measurements. 


\section{FRAP assay}

The ferric-reducing ability of plasma (FRAP assay) was adapted from the original protocol (Benzie and Strain 1996) with minor modifications introduced by Pulido and colleagues (2000). This method is based on a single electron transfer reaction between an oxidant and the antioxidants, ie, plasma antioxidants are oxidized by the oxidant Fe(III). Thus, a single electron is transferred from the antioxidant molecule to the oxidant. Briefly, $900 \mu \mathrm{L}$ of FRAP reagent, prepared freshly was mixed with $90 \mu \mathrm{L}$ of distilled water and $30 \mu \mathrm{L}$ of plasma sample. Water was used for the reagent blank. The FRAP reagent contained $2.5 \mathrm{~mL}$ of a $10 \mathrm{mmol} / \mathrm{L} \mathrm{TPTZ}$ solution in $40 \mathrm{mmol} / \mathrm{L} \mathrm{HCl}$ plus $2.5 \mathrm{~mL}$ of $20 \mathrm{mmol} / \mathrm{L} \mathrm{FeCl} 3 * 6 \mathrm{H} 2 \mathrm{O}$ and $25 \mathrm{~mL}$ of $0.1 \mathrm{~mol} / \mathrm{L}$ acetate buffer, $\mathrm{pH} 3.6$. The change of absorbance of the Fe-(TPTZ)2(III) complex (FRAP reagent), due to the action of an antioxidant in the plasma, was measured at $593 \mathrm{~nm}$ by a spectrophotometer after twenty minutes of reaction-stabilization period. The absorbance value is used as the measurement for the reducing capability of the antioxidant. Aqueous solutions of known Fe(II) concentrations in the range of $100-2000 \mu \mathrm{mol} / \mathrm{L}$ ( $\mathrm{FeSO} 4 * 7 \mathrm{H} 2 \mathrm{O}$ ) were used for calibration. All measurements were performed in triplicates. FRAP analysis is widely used in the evaluation of antioxidant capacity in biological fluids including plasma, since it is a simple, reliable, and fast technique. FRAP measurements were complemented with TAC-crocin that allows determinations of the protecting capacity of blood plasma against peroxidative agents, as indicated below.

\section{Serum lipid peroxide measurement}

Levels of lipid peroxidation were measured as the formation of a thiobarbituric acid (TBA) adduct of malondialdehyde (MDA). The serum lipid peroxide measurement was used with minor modifications in the original protocol (Satoh 1978). The standard procedure was established as follows: To $0.25 \mathrm{ml}$ of plasma sample, $1.25 \mathrm{ml}$ of $1.23 \mathrm{M}$ trichloroacetic acid (TCA) were added and the mixture was left to react for $10 \mathrm{~min}$ at room temperature. After centrifugation at $1500 \mathrm{~g}$ for $10 \mathrm{~min}$, the supernatant was decanted and $1.25 \mathrm{~mL}$ of sulfuric acid $5 \mathrm{mM}$ was aggregated to the precipitate, and then ultrasonicated three times for $15 \mathrm{sec}$ immersion in icecool water. Next, we added $1.5 \mathrm{~mL}$ of TBA $16 \mathrm{mM}$, and the coupling reaction of the lipid peroxide with TBA was carried out by heating in a boiling water bath for $60 \mathrm{~min}$. After cooling in cold water, the resulting chromogen was extracted with $1.0 \mathrm{ml}$ of $\mathrm{n}$-butanol by vigorous shaking in vortex. The organic phase was separated by centrifugation at $1500 \mathrm{~g}$ for $5 \mathrm{~min}$. Then, the optical density was determined at $532 \mathrm{~nm}$.
The standard curves were performed with MDA solutions $(0.075-2.25 \mu \mathrm{mol} / \mathrm{L})$, and $5 \mathrm{mM}$ sulfuric acid was used as a blank. All measurements were performed by triplicate.

\section{Total antioxidant capacity by the crocin bleaching assay}

Crocin bleaching assay was used with minor modifications to the original protocols (Tubaro et al 1996; Malliaraki et al 2003). Briefly, crocin was extracted and purified from saffron according with the method of Ordoudi and Tsimidou (2006). A stock solution of crocin was prepared in methanol and was diluted with $0.1 \mathrm{~mol} \mathrm{L-1}$ phosphate buffer, $\mathrm{pH} 7.0$ in order to obtain a $1.35 \times 10^{-5} \mathrm{~mol} \mathrm{~L}-1$ crocin solution (the absorption coefficient of crocin at $443 \mathrm{~nm}$ is $1.33 \times 105 \mathrm{~mol}-1 \mathrm{~cm}-1)$. As initiator, a stock solution of AAPH (250 mmol L-1) was prepared in PBS $10 \mathrm{mmol} \mathrm{L-1} \mathrm{pH}$ 7.0. The assay was performed in the cuvettes adding the reactants in the following order: $20 \mu \mathrm{L}$ of plasma sample or standard, $80 \mu \mathrm{L}$ of AAPH (250 mmol L-1) and finally $1000 \mu \mathrm{L}$ of crocin solution. Bleaching rate of crocin at $443 \mathrm{~nm}$ was monitored at $40{ }^{\circ} \mathrm{C}$ during 25 minutes using a V-500 spectrophotometer (Jasco, Easton, MD, USA). The blank reference cuvette contained only phosphate buffer solution. Antioxidant capacity was calculated using similar expressions adapted from ORAC methodology (Cao et al 1999; Ou et al 2001). The corrected area under the relative absorbance curve (AUC) of each sample was compared with those obtained from the standard curve made from Trolox (3.5-60 $\mu \mathrm{mol} \mathrm{L-1)}$, and results were expressed as $\mu \mathrm{mol}$ of Trolox equivalent per milliliter of plasma (TEAC). These TEAC values refer to the net protection (AUC) of crocin in presence of an antioxidant. All measurements were performed in triplicates. Thus, in all the studies corrected values of TAC were obtained after substracting circularing antioxidant agents such as uric acid, bilirubin, and lipoproteins (Kampa et al 2002).

\section{Statistical analysis}

Statistical analysis was performed with GraphPad Prism4 program (GraphPad Software, La Jolla, CA, USA). Comparisons were made by student $t$-test for normally distributed variables. One-way ANOVA and Bonferroni's Multiple Comparison Test were used to test differences in mean values. All contrasts were made considering alpha lower than $5 \%$ to be an error. Correlations were assessed by Pearson's method.

\section{Results}

Demographic and neuropsychological data among the two groups in the study are given in Table 1 . No significant 
Table I Demographic data and cognitive performance differences between groups in the study

\begin{tabular}{|c|c|c|c|c|c|c|c|}
\hline & \multicolumn{3}{|c|}{ Control group } & \multicolumn{4}{|c|}{ AD group } \\
\hline & $\bar{n}$ & Mean value & SD & $\mathrm{n}$ & Mean Value & SD & $\mathbf{p}$ \\
\hline Age & 29 & 70.7 & 6.9 & 59 & 76.4 & 6.1 & $\leq 0.05$ \\
\hline Education & 29 & 8.6 & 3.0 & 59 & 7.7 & 4.5 & $\geq 0.05$ \\
\hline MMSE & 29 & 25.0 & 3.1 & 53 & $\mid 4 . I^{*}$ & 6.0 & $\leq 0.05$ \\
\hline PRAXIAS & 29 & 4.8 & 0.7 & 53 & $3.4^{*}$ & 1.6 & $\leq 0.05$ \\
\hline FLU ANIMAL & 29 & 14.4 & 4.2 & 48 & $7.4^{*}$ & 3.5 & $\leq 0.05$ \\
\hline FLU A & 28 & 9.4 & 3.7 & 45 & $5.0^{*}$ & 3.2 & $\leq 0.05$ \\
\hline BNT & 28 & 10.0 & 2.6 & 42 & $4.7^{*}$ & 4.0 & $\leq 0.05$ \\
\hline FAB & 27 & 13.8 & 3.0 & 43 & $9.0 *$ & 3.4 & $\leq 0.05$ \\
\hline TAM & 29 & 39.7 & 5.6 & 37 & $16.9 *$ & 8.2 & $\leq 0.05$ \\
\hline
\end{tabular}

Abbreviations: AD, Alzheimer's disease; BNT, Boston Naming Test; FAB, Frontal Assessment Battery; FLU A, semantic verbal fluency of words with initial A; FLU ANIMAL, Semantic Verbal Fluency of animals with initial A; MMSE, Mini Mental State Examination; PRAXIAS, Ideomotor Praxia by imitation; SD, standard deviation; TAM, Test of Metalinguistic Ability.

differences were found in the educational levels between the subsets of subjects $(p>0.05)$. A significant difference $(p=0.0002)$ in the mean ages of AD patients as compared with ages of the control group was obtained in the recruited population. AD patients were from moderately to significantly impaired in their cognitive performance, on the basis of all neuropsychological tests relative to control performance. As expected, the means of $\mathrm{AD}$ group were smaller than the control group in all the tests.

Mean values of cholesterol parameters and SD are reported in Table 2. No significant differences were found between the two AD and control subsets in the study. This data also agreed with other authors (Corzo et al 2007).

The oxidative stress biomarkers analyzed in this article are summarized in Table 3. In all the assays applied, no statistical differences were observed between the AD subset and the control subjects. However, when subjects exhibiting cognitive impairment were divided into subgroups according with their GDS performance score, significant differences in FRAP were found between the controls and the moderate and severe AD subgroups, and between mild and moderate/ severe AD (Figure 1).

Table 2 Comparison of the cholesterol parameters $(\mathrm{mg} / \mathrm{dL})$ of AD subjects and neurologically normal controls

\begin{tabular}{llllllll}
\hline & \multicolumn{2}{l}{ Control $(\mathbf{n}=\mathbf{2 9})$} & & AD $(\mathbf{n}=\mathbf{5 5})$ & \\
\cline { 2 - 3 } \cline { 6 - 7 } & Mean Value & SD & & Mean Value & SD & p value \\
\hline Total-cholesterol & 200 & 42 & 202 & 39 & $>\mathbf{0 . 0 5}$ \\
Tryglicerides & 147 & 58 & 132 & 55 & $>\mathbf{0 . 0 5}$ \\
HDL-cholesterol & 54 & 13 & 52 & 11 & $>\mathbf{0 . 0 5}$ \\
LDL-cholesterol & 122 & 34 & 127 & 34 & $>\mathbf{0 . 0 5}$ \\
VLDL-cholesterol & 23 & 7 & 23 & 8.0 & $>\mathbf{0 . 0 5}$ \\
cholest/HDL index & 3.8 & 0.7 & 4.0 & 0.9 & $>\mathbf{0 . 0 5}$ \\
\hline
\end{tabular}

Abbreviations: AD, Alzheimer's disease; HDL, high-density lipoprotein; LDL, low-density lipoprotein; SD, standard deviation
However, differences between $\mathrm{AD}$ and controls were not observed for the MDA levels and TAC-crocin when the subgroups were classified by GDS scores. Then, we tried to find correlations between the cognitive performance and oxidative stress biomarkers. Antioxidant capacity values measured by FRAP methods were positively correlated with MMSE $(\mathrm{r}=0.443, \mathrm{P}<0.01)$ and PRAXIAS $(\mathrm{r}=0.301, \mathrm{P}<0.05)$ (Figure 2). We could not find another correlation between oxidative stress status and the cognitive impairment.

Finally, we investigated the correlations between cholesterol parameters and the results of the neuropsychological tests. In this case, a negative correlations between total cholesterol $(\mathrm{r}=-0.298, \mathrm{P}<0.05)$ and VLDL $(\mathrm{r}=-0.382, \mathrm{P}<0.01)$ versus GDS was found (Figure 3$)$. Instead, a positive correlation $(\mathrm{r}=0.464, \mathrm{P}<0.01)$ was found between the cholesterol/HDL index and the BNT or semantic memory (Figure 4). No correlations were found with the other cholesterol metabolism parameters like HDL, LDL, or triglycerides.

\section{Discussion}

We did not find statistically significant differences in determinations of plasma FRAP and TAC-crocin in AD subjects as compared with controls. Similar results are reported in the literature concerning plasma TAC measurement in AD patients (Sinclair et al 1998; Feillet-Coudray et al 1999; Pulido et al 2005), however other studies have shown a lower plasma TAC in AD patients (Reppetto et al 1999; Serra et al 2001; Guidi et al 2006).

However, when the subset of cognitively impaired subjects were stratified by their GDS status, significant differences were found in the FRAP average values between the subgroups of patients. In our study, those patients with moderate/severe $\mathrm{AD}$ displayed the lowest anti-oxidant capacity 
Table 3 FRAP, MDA levels, and TAC-crocin mean values and SD in both AD and control groups

\begin{tabular}{|c|c|c|c|c|c|c|c|}
\hline & \multicolumn{3}{|c|}{ Control } & \multicolumn{4}{|c|}{ AD } \\
\hline & n & Mean value & $\overline{S D}$ & $\mathbf{n}$ & Mean value & SD & $P$ value \\
\hline$\overline{\text { FRAP }\left(\mu \mathrm{M} \text { eq. } \mathrm{Fe}^{2+}\right)}$ & 29 & 1,215 & 317 & 59 & 1,118 & 253 & $>0.05$ \\
\hline MDA levels $(\mu \mathrm{mol} / \mathrm{L})$ & 29 & 0.67 & 0.48 & 59 & 0.57 & 0.34 & $>0.05$ \\
\hline TAC-crocin ( $\mu \mathrm{mol} / \mathrm{L}$ Trolox/mL plasma) & 14 & 13.1 & 6.6 & 31 & 11.2 & 4.8 & $>0.05$ \\
\hline
\end{tabular}

Abbreviations: AD, Alzheimer's disease; FRAP, ferric-reducing ability of plasma; MDA, malondialdehyde; SD, standard deviation; TAC, total antioxidative capacity.

by FRAP assay. This result is consistent with Zafrilla and colleagues (2006) that characterized the total antioxidant plasmatic status of the $\mathrm{AD}$ patients. Both the light-moderate $\mathrm{AD}$ subjects and patients with an advanced phase of $\mathrm{AD}$ exhibited lower FRAP values than in the controls. This is a novel and interesting observation. Previous reports that investigated this specific aspects, did not find this type of relationship in the plasma of $\mathrm{AD}$ patients (Ceballos-Picot et al 1996). Interestingly, Pulido and colleagues (2005) discovered a lower antioxidant capacity of AD plasma samples by FRAP method only in subjects with APOE genotype $\varepsilon 4 / \varepsilon 4$, which is the group with the highest incidence of $\mathrm{AD}$. We have to point out that FRAP should be analyzed in a global context together with other biochemical parameters, and considering some nutritional conditions of subjects.

On the other hand, we observed no modification in plasma malondialdehyde (MDA) levels when AD patients were compared with control subjects. This observation is different from previous publications that reported significant differences in MDA levels of erytrocytes of AD patients as compared with the controls (Bermejo et al 1997). In other studies, the ex - vivo susceptibility to lipid peroxidation was higher in mild and moderate $\mathrm{AD}$ with respect to controls, but not in

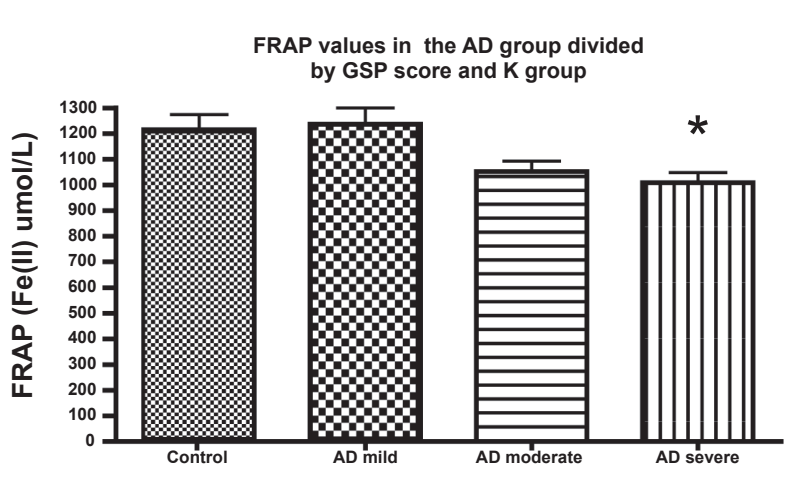

Figure I Ferric reducing ability of plasma (eq. Fe(II) $\mu \mathrm{M}$ ) of patients with Alzheimer's disease at different stages of the disease, as evaluated by GDS. Other details in Methods. The number of subjects in every group $(n)$ was: $n=23$ in the mild AD (GDS $=3-4) ; n=24$ in the moderate $A D$ (GDS 5), and $n=12$ for severe $A D ; n=29$ for the control group. Note: * Significant differences $(p<0.05$ ) between control subgroup (or mild $A D$ ) vs moderate or severe AD. One-way ANOVA and Bonferroni's comparison test wre used to assess differences in mean values. severe AD patients (Galbusera et al 2004). Peroxidation was higher in patients in the advanced stage of the disease than in the control group. However, no significant differences were observed between the different stages of the disease (Zafrilla et al 2006).

We explored the association of plasma oxidative components to cognitive impairment in mild and severe conditions
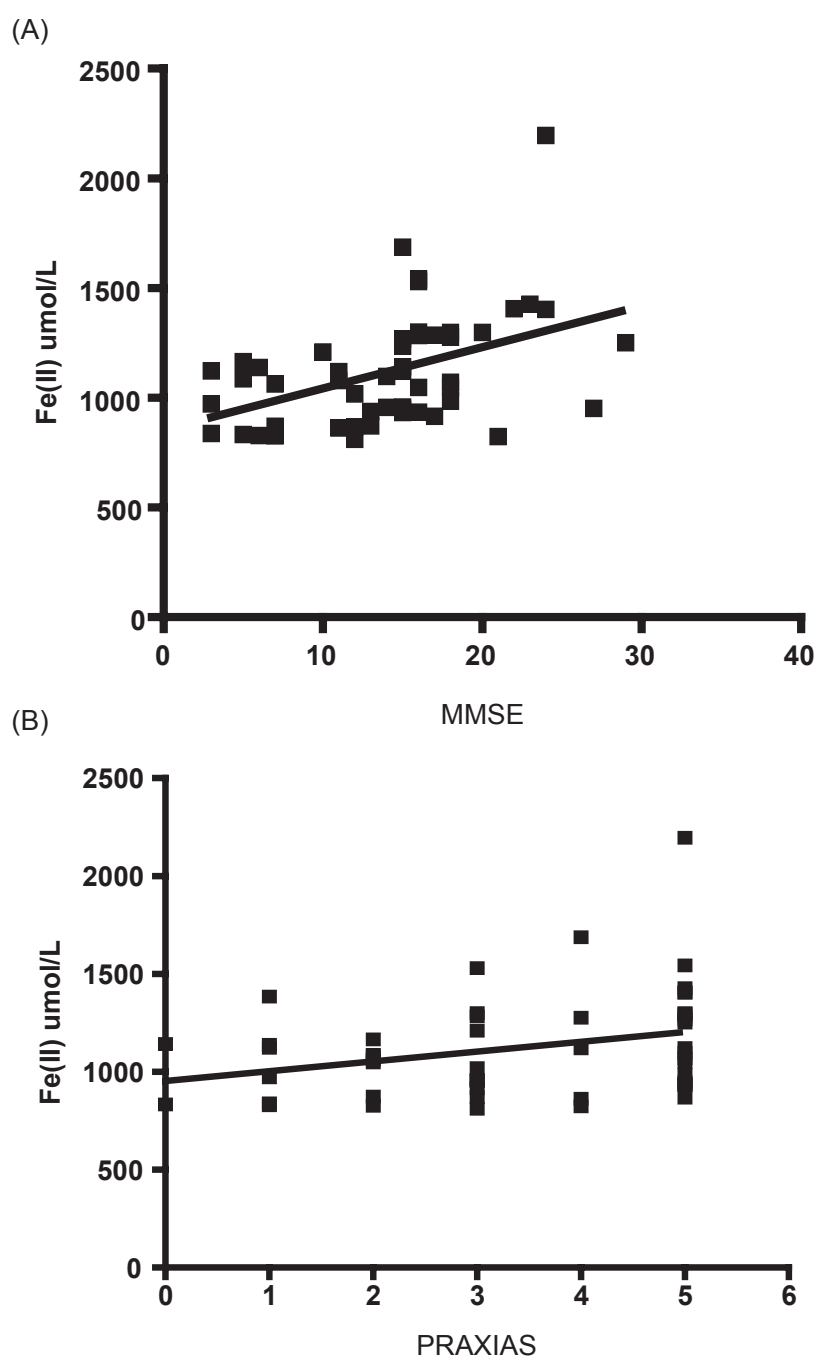

Figure 2 (A) Correlation between FRAP (eq Fe(II) $\mu \mathrm{M}$ ) and cognitive impairment in AD patients $(r=0.443, P<0.01)$. (B) Correlation between FRAP (eq Fe(II) $\mu \mathrm{M})$ and PRAXIAS test $(r=0.30 I, P<0.05)$. 


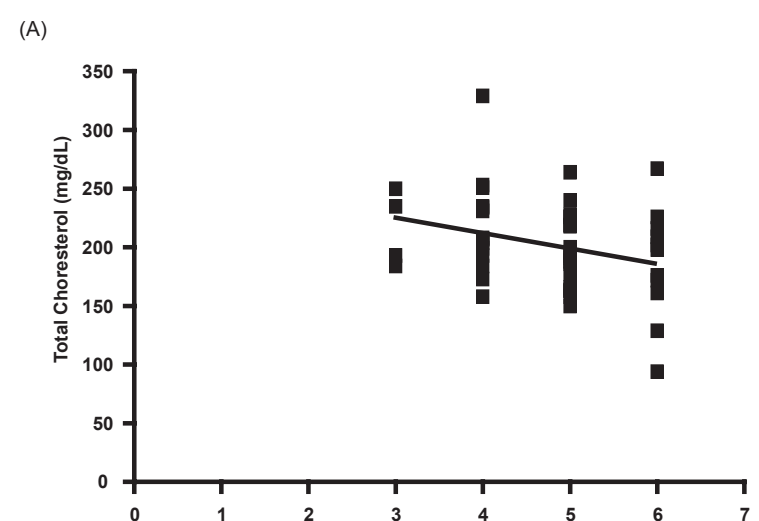

(B)

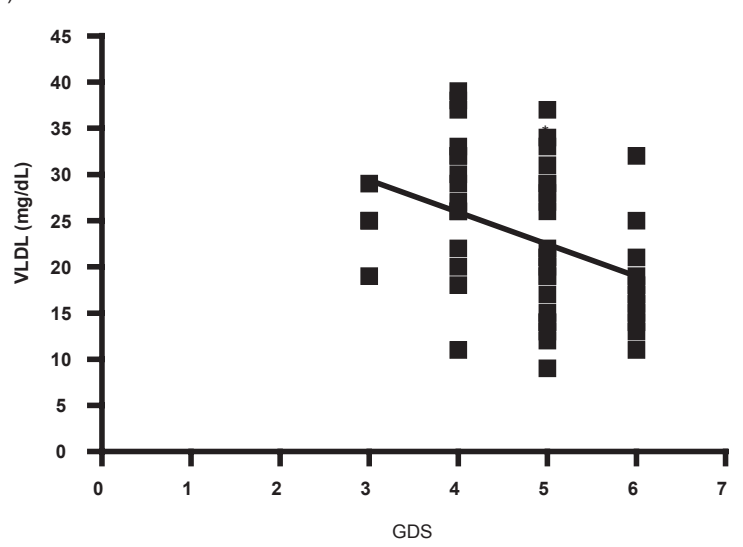

Figure 3 (A) Correlation between total cholesterol ( $\mathrm{mg} / \mathrm{dL}$ ) and GDS in AD patients $(r=-0.298, P<0.05)$. (B) Correlation between VLDL $(\mathrm{mg} / \mathrm{dL})$ and GDS in AD patients $(r=-0.382, P<0.01)$.

of AD. We found that cognitive impairment parameters MMSE and PRAXIAS were both positively correlated with the FRAP, this results indicates that oxidative damage in demented patientes can be promoted in part by a reduced ability of plasma to scavenge free radicals. Interestingly, in a previous report it has been suggested that a decrease in TAC of plasma is negatively correlated with the duration of moderate/severe AD and mild AD, and significant increases in plasma total homocysteine are also present in AD patients (tHcy) (Guidi et al 2006).

Previous studies on the links between plasma levels of lipids and cholesterol with AD exihibit a marked controversy. When we explored the association of plasma lipids to cognitive impairment in these groups we found no significant differences in LDL, HDL, VLDL and total cholesterol levels between AD patients and healthy controls. This is consistent with recent reports decribing no association between lipids and the risk of amnesiac or nonamnesiac mild AD (Reitz et al 2008). However, we found a slightly negative correlation between GDS against total cholesterol,

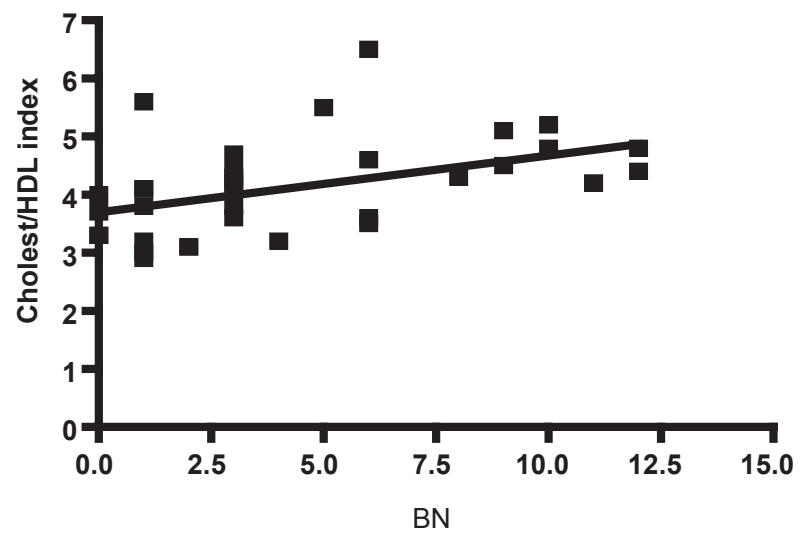

Figure 4 Correlations between Cholesterol/HDL index and Boston Naming Test (BNT) values in AD patients $(r=0.464, P<0.01)$. Other experimental details in Methods section.

also GDS status against VLDL levels in AD group respect to controls. These clinical data may seem controversial, in the light of the current hypothesis that links cholesterol levels with amyloid pathology and tau hyperphosphorylation based on in vitro studies (Rojo et al 2006), but they are consistent with recent reports describing the lipid profile of demented patients (Dimopoulos et al 2007). An important contribution of our work to elucidate the association of lipids with cognitive impairment is the finding of a positive correlation between the cholesterol/HDL ratio and the impairment of semantic memory (BNT test) (Figure 4). This is a very interesting finding in the light of recent reports decribing correlations between metabolic syndrome and high risk of cognitive decline (Komulainen et al 2007; Panza et al 2007).

Studies of the changes in cholesterol levels have shown that thotal cholesterol tends to increase with age in young or middle-aged adults but later decreases as individuals get older (Abbott et al 1997; Ferrara et al 1997). Another study shows that total cholesterol levels in plasma were lower in patients with $\mathrm{AD}$ than in controls at least five years before diagnosis (Mielke et al 2005), and decreased more rapidly among people who subsequently developed dementia (Notkola et al 1998). Solomon and colleagues (2007) have proposed that high midlife serum TC is a risk factor for subsequent dementia/AD, but decreasing serum total cholesterol after midlife may reflect ongoing disease processes and may represent a risk marker for late-life cognitive impairment. Apparently, several years before clinical evidence of dementia, total cholesterol begins to decline, possibly as a results of the ongoing AD pathology (Panza et al 2007). On the other hand, the decrease in plasma total 
cholesterol in the development of disease may be partially explained by the physiological aging process. However, no correlation was evidenced between age and the total cholesterol, VLD-cholesterol and cholesterol/HDL index in the AD patients under our study. The minor decrease detected with the time progression of $\mathrm{AD}$ might be explained by unintentional or voluntary changes in lifestyle-related factors because of the reduced quality-of-life of AD patients.

In summary, in a study on human subjects we corroborated the findings that the degree of oxidative stress is directly related to the advance of cognitive impairment and AD. Thus, high correlations between the oxidative stress marker FRAP and cognitive impairment indicate that oxidative stress should be considered a risk factor in the development of cognitive disorders (Perry et al 2000). Oxidative stress is critical in the pathogenesis of $\mathrm{AD}$, and is involved in biological damage, thus one might think that the administration of antioxidants may be useful in the prevention and treatment of $\mathrm{AD}$ and other neurodegenerative disorders.

In the meantime, we demonstrated that plasmatic levels of cholesterol also decrease with the progression of dementia in the AD population as evaluated by changes in GDS measurements (Figure 3). This might be a result of the pathologic processes leading to dementia, at least during the early stages of the disease. The negative correlations between cholesterol parameters and the cognitive impairment open new questions about the role of cholesterol and oxidative markers in AD progression. Interestingly, in a recent study on the levels of brain redox-iron overload, a positive correlation between cerebral spinal fluid redox-iron and AD progression was evidenced at the early stages of the disease (mild AD cases), but this particular iron compartment decreased at advanced stages of the disease (Lavados et al 2008).

\section{Acknowledgments}

Research was supported by grants 1050198 and 1080254 from FONDECYT, the International Center for Biomedicine (to RBM) and by the Bicentennial Project IPC-79 of PBCT-CONICYT (to AS). The authors report no conflicts of interest in this work.

\section{References}

Abbott RD, Sharp DS, Burchfield CM, et al. 1997. Cross-sectional and longitudinal changes in total and high-density-lipoprotein cholesterol levels over a 20-year period in elderly men: the Honolulu Heart Program. Ann Epidemiol, 7:417-24.

Barnham KJ, Masters CL, Bush AI. 2004. Neurodegenerative diseases and oxidative stress. Nat Rev Drug Discov, 3:205-14.

Benzie IFF, Strain JJ. 1996. The ferric reducing ability of plasma (FRAP) as a measure of "antioxidant power": the FRAP assay. Anal Biochem, 239:70-6.
Bermejo P, Gómez-Serranillos P, Santos J, et al. 1997. Determination of malonaldehyde in Alzheimer's disease: a comparative study of high-performance liquid chromatography and thiobarbituric acid test. Gerontology, 43:218-22.

Berr C, Balansard B, Arnaud J, et al. 2000. Cognitive decline is associated with systemic oxidative stress: the EVA study, Etude du Vieillissement Arteirel. J Am Geriatr Soc, 48:1285-91.

Cao G, Prior R. 1999. Measurement of oxygen radical absorbance capacity in biological samples. Oxidants and antioxidants. Methods Enzymol, 299:50-62.

Ceballos-Picot I, Mansouria MB, Nicole A, et al. 1996. Peripheral antioxidant enzyme activities and selenium in elderly subjects and in dementia of Alzheimer's type place of the extracellular glutathione peroxidase. Free Radical Biol Med, 20:579-87.

Corder EH, Saunders AM, Strittmatter WJ, et al. 1993. Gene dose of apolipoprotein E type 4 allele and the risk of Alzheimer's disease in late onset families. Science, 261:921-3.

Corzo L, Zas R, Rodríguez S, et al. 2007. Decreased levels of nitric oxide in different forms od dementia. Neurosci Lett, 420:263-7.

Dimopoulos N, Piperi C, Salonicioti A, et al. 2007. Characterization of the lipid profile in dementia and depression in the elderly. $J$ Geriatr Psychiatry Neurol, 20:138-44.

Feillet-Coudray C, Tourtauchaux R, Niculescu M, et al. 1999. Plasma levels of 8-epiPGF2a, an in vivo marker of oxidative stress, are not affected by aging or Alzheimer's disease. Free Radic Biol Med, 27:463-9.

Ferrara A, Barrett-Connor E, Shan J. 1997. Total, LDL, and HDL cholesterol decrease with age in older men and women. The Rancho Bernardo Study 1984-1994. Circulation, 96:37-43.

Galbusera C, Facheris M, Magni F, et al. 2004. Increased susceptibility to plasma lipid peroxidation in Alzheimer's disease patients. Curr Alzheimer Res, 1:103-9.

Goedert M, Spillantini MG. 2006. A century of Alzheimer's disease. Science, 314:777-81.

Guidi I, Galimberti D, Lonati S, et al. 2006. Oxidative imbalance in patients with mild cognitive impairment and Alzheimer's Disease. Neurobiol Aging, 27:262-9.

Kampa M, Nistikaki A, Tsausis V, et al. 2002. A new authomated method for determination of the total antioxidant capacity (TAC) of human plasma based on crocin bleaching assay. BMC Clin Pathol, 2:1-16.

Kikuchi A, Takeda A, Onodera H, et al. 2002. Systemic increase of oxidative nucleic acid damage in Parkinson's disease and multiple atrophy. Neurobiol Dis, 9:44-8.

Kivipelto M, Helkala EL, Laakso MP, et al. 2001. Midlife vascular risk factors and Alzheimer's disease in later life: longitudinal, population based study. BMJ, 322:1447-51.

Komulainen P, Lakka TA, Kivipelto M, et al. 2007. Metabolic syndrome and cognitive function: a population-based follow-up study in elderly women. Dement Geriatr Cogn Disord, 23:29-34.

Lavados M, Farias G, Rothhammer F, et al. 2005. ApoE alleles and tau markers in pateints with different levels of cognitive impairment. Arch Med Res, 36:474-9.

Lavados M, Guillon M, Mujica MC, et al. 2008. Mild cognitive impairment and Alzheimer's patients disk'lay different levels of redox CSF iron. J Alz Dis, 13:225-32.

Leininger-Muller B, Jolivalt C, Bertrand P, et al. 1998. Oxidation of human apolipoprotein E: isoforms susceptibility and protection with Ginkgo biloba EGb 761 extract. In: Packer L, Christen Y, (eds). Ginkgo biloba Extract (Egb761) Study: Lesson from Cell Biology. Elsevier, Paris, pp. 57-68.

Maccioni RB, Munoz JP, Barbeito L. 2001. The molecular bases of Alzheimer's disease and other neurodegenerative disorders. Arch Med Res, 32:367-81.

Malliaraki N, Mpliamplias D, Kampa M, et al. 2003. Total and corrected antioxidant capacity in hemodialyzed patients. BMC Nephrol, 4:4.

McKhann G, Drachman D, Folstein M, et al. 1984. Clinical diagnosis of Alzheimer's disease: Report of the NINCDS-ADRDA work group under the auspices of the Department Of Health and Human Services Task Force on Alzheimer's Disease. Neurology, 34:939-44. 
Mielke MM, Zandi PP, Sjogren M, et al. 2005. High total cholesterol levels in late-life associated with a reduced risk of dementia. Neurology, 64:1689-95.

Notkola IL, Sulkava R, Pekkanen J, et al. 1998. Serum total cholesterol, apolipoprotein E epsilon 4 allele, and Alzheimer's disease. Neuroepidemiology, 17:14-20.

Nunomura A, Moreira PI, Lee HG, et al. 2007. Neuronal death and survival under oxidative stress in Alzheimer disease. CNS Neurol Dis Drug Targets, 6:411-23.

Ordoudi SA, Tsimidou MZ. 2006. Crocin bleaching assay step by step: observations and suggestions for an alternative validated protocol. $J$ Agric Food Chem, 5:1663-71.

Ou B, Hampsch-Woodill M, Prior R. 2001. Development and validation of an improved oxygen radical absorbance capacity assay using fluorescein as the fluorescent probe. J Agric Food Chem, 49:4619-26.

Panza F, Capurso C, D'Introno A, et al. 2007. Total cholesterol levels and the risk of mild cognitive impairment and Alzheimer's disease. $J$ Am Geriatr Soc, 55:133-5.

Perry G, Nunomura A, Jones PK, et al. 2000. Oxidative imbalance is a major feature of Alzheimer disease. Curr Biochem Res, 3:151-6.

Pulido R, Bravo L, Saura-Calixto F. 2000. Antioxidant activity of dietary polyphenols as determined by a modified ferric reducing/antioxidant power assay. J Agric Food Chem, 48:3396-402.

Pulido R, Jiménez-Escrig A, Orensanz L, et al. 2005. Study of plasma antioxidant status in Alzheimer's disease. Eur J Neurol, 12:531-5.

Ramassamy C, Krzywokowski P, Bastianetto S, et al. 1998. Apolipoprotein E, oxidative stress and EGb 761 in Alzheimer's disease brain. In: Packer L, Christen Y, (eds). Ginkgo biloba Extract (EGb 761) Study: Lesson from Cell Biology. Paris: Elsevier, pp. 69-83.
Reisberg B, Franssen EH, Souren LE, et al. 1998. Progression of Alzheimer's disease: variability and consistency: ontogenic models, their applicability and relevance. J Neural Transm Suppl, 54:9-20.

Reitz C, Tang MX, Manly J, et al. 2008. Plasma lipid levels in the elderly are not associated with the risk of mild cognitive impairment. Dement Geriatr Cogn Disord, 25:232-7.

Repetto MG, Reides CG, Evelson P et al. 1999. Peripheral markers of oxidative stress in probable Alzheimer patients (1999). Eur J Clin Invest, 29:643-9.

Rojo L, Sjöberg MK, Hernández P, et al. 2006. Roles of cholesterol and lipids in the etiopathogenesis of Alzheimer's disease. J Biomed Biotechnol, 2006:73976.

Satoh K. 1978. Serum lipid peroxide in cerebrovascular disorders determined by a new colorimetric method. Clin Chim Acta, 90:37-43.

Serra JA, Dominguez RO, de Lustig ES, et al. 2001. Parkinson's disease is associated with oxidative stress: comparison of peripheral antioxidant proles in living Parkinson's, Alzheimer's and vascular dementia patients. J Neural Transm, 108:1135-48.

Sinclair AJ, Bayer A, Johston JO, et al. 1998. Altered plasma antioxidant status in subjects with Alzheimer's disease and vascular dementia. Int $J$ Geriat Psychiatry, 13:840-5.

Solomon A, Kåreholt I, Ngandu T, et al. 2007. Serum cholesterol changes after midlife and late-life cognition: Twenty-one-year follow-up study. Neurology, 68:751-6.

Tubaro F, Micossi E, Ursini F. 1996. The antioxidant capacity of complex mixtures by kinetic analysis of crocin bleaching inhibition. $J$ Am Oil Chem Soc, 73:173-9.

Zafrilla P, Mulcro J, Xandri JM, et al. 2006. Oxidative stress in Alzheimer patients in differents stages of the disease. Curr Med Chem, 13:1075-83. 